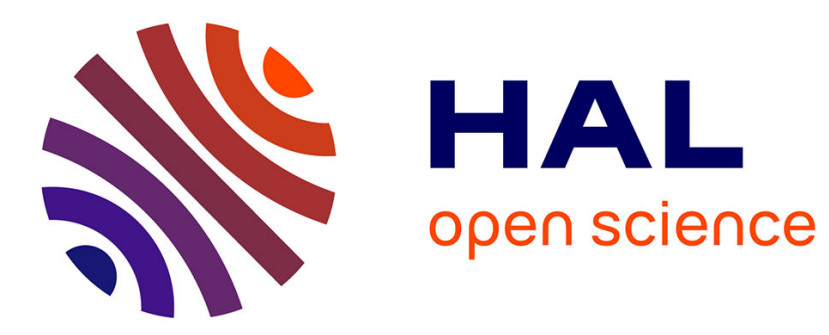

\title{
A Theorem of Barany Revisited and Extended
}

\author{
Nabil Mustafa, Ray Saurabh
}

\section{To cite this version:}

Nabil Mustafa, Ray Saurabh. A Theorem of Barany Revisited and Extended. 2012 Symposium on

Computational Geometry, Jun 2012, United States. pp.333-338. hal-00761355

\section{HAL Id: hal-00761355 \\ https://hal.science/hal-00761355}

Submitted on 5 Dec 2012

HAL is a multi-disciplinary open access archive for the deposit and dissemination of scientific research documents, whether they are published or not. The documents may come from teaching and research institutions in France or abroad, or from public or private research centers.
L'archive ouverte pluridisciplinaire HAL, est destinée au dépôt et à la diffusion de documents scientifiques de niveau recherche, publiés ou non, émanant des établissements d'enseignement et de recherche français ou étrangers, des laboratoires publics ou privés. 


\section{A Theorem of Bárány Revisited and Extended}

\author{
Nabil H. Mustafa \\ Department of Computer Science \\ Université Paris-Est, ESIEE Paris, 93162 \\ Noisy-Le-Grand Cedex, France \\ mustafan@esiee.fr
}

\author{
Saurabh Ray \\ Max-Planck-Institut für Informatik \\ 66123 Saarbrücken, Germany \\ saurabh@mpi-inf.mpg.de
}

\begin{abstract}
The colorful Carathéodory theorem [Bár82] states that given $d+1$ sets of points in $\mathbb{R}^{d}$, the convex hull of each containing the origin, there exists a simplex (called a 'rainbow simplex') with at most one point from each point set, which also contains the origin. Equivalently, either there is a hyperplane separating one of these $d+1$ sets of points from the origin, or there exists a rainbow simplex containing the origin. One of our results is the following extension of the colorful Carathéodory theorem: given $\lfloor d / 2\rfloor+1$ sets of points in $\mathbb{R}^{d}$, and a convex object $C$, then either one set can be separated from $C$ by a constant (depending only on $d$ ) number of hyperplanes, or there is a $\lfloor d / 2\rfloor$-dimensional rainbow simplex intersecting $C$.
\end{abstract}

\section{Categories and Subject Descriptors}

G.2 [Combinatorics]: Discrete Geometry

\section{General Terms}

Algorithms, Theory

\section{Keywords}

Discrete Geometry, Weak $\epsilon$-nets, Caratheodory's theorem, Colorful Caratheodory theorem, Hadwiger-Debrunner $(p, q)$-theorem

\section{INTRODUCTION}

The goal of this paper is to study the behavior of low-dimensional simplices with respect to convex sets in $\mathbb{R}^{d}$. We examine a number of classical theorems in discrete geometry - Radon's theorem [Rad47], Carathéodory's theorem [Mat02], colorful Carathéodory theorem [Bár82] - and prove extensions that demonstrate the phenomenon of low-dimensional intersections.

\section{Three classical theorems.}

One of the starting theorems in discrete geometry is the following result. For a set $P \subset \mathbb{R}^{d}$, let $\operatorname{conv}(P)$ denote the convex hull of $P$.

Permission to make digital or hard copies of all or part of this work for personal or classroom use is granted without fee provided that copies are not made or distributed for profit or commercial advantage and that copies bear this notice and the full citation on the first page. To copy otherwise, to republish, to post on servers or to redistribute to lists, requires prior specific permission and/or a fee.

SCG'12, June 17-20, 2012, Chapel Hill, North Carolina, USA.

Copyright 2012 ACM 978-1-4503-1299-8/12/06 ...\$10.00.
THEOREM 1 (RADON's THEOREM). Given any set $P$ of $d+$ 2 points in $\mathbb{R}^{d}$, one can partition $P$ into two sets $P_{1}$ and $P_{2}$ such that $\operatorname{conv}\left(P_{1}\right) \cap \operatorname{conv}\left(P_{2}\right) \neq \emptyset$.

Note here that one of the two sets $P_{1}$ and $P_{2}$ can be large, e.g., $P_{1}$ can consist of $d+1$ points. So only the trivial bound $\left|P_{1}\right|,\left|P_{2}\right| \leq$ $d+1$ holds. Therefore one cannot get a better upper bound on the dimension of the simplices $\operatorname{conv}\left(P_{1}\right)$ or $\operatorname{conv}\left(P_{2}\right)$.

We say a point $p$ can be separated from a convex set $C$ if there exists a hyperplane $h$ with $C$ and $p$ in the interior of the two different halfspaces defined by $h$.

THEOREM 2 (CARATHÉODORY'S THEOREM). If a convex set $C$ intersects the convex hull of some point set $P$, then it also intersects a simplex spanned by $P$. Equivalently, either $P$ can be separated from $C$ with one hyperplane, or $C$ intersects the convex hull of some $(d+1)$ points of $P$.

Here we have stated the theorem in a slightly more general form; usually it is stated where $C$ is just a point.

A beautiful extension of Carathéodory's theorem was discovered by Imre Bárány [Bár82]:

Theorem 3 (COLORFul CARATHÉOdory THEOREM). Given $d+1$ sets of points $P_{1}, \ldots, P_{d+1}$ in $\mathbb{R}^{d}$ and a convex set $C$ such that $C \cap \operatorname{conv}\left(P_{i}\right) \neq \emptyset$ for all $i=1, \ldots, d+1$, there exists a set $Q$ with $C \cap \operatorname{conv}(Q) \neq \emptyset$ and where $\left|Q \cap P_{i}\right|=1$ for all $i$. Such a $Q$ is called a 'rainbow set'. Equivalently, either some $P_{i}$ can be separated from $C$ with one hyperplane, or $C$ intersects the convex hull of a rainbow set of $d+1$ points.

This theorem is also commonly stated for the case where $C$ is a point, but the above slight generalization follows immediately from Bárány's proof technique [Bár82]. Also, Carathéodory's theorem follows by applying the colorful Carathéodory theorem to $d+1$ copies of the same pointset.

\section{Our results.}

The starting point of our work is the following well-known generalization of the Erdös-Szekeres theorem:

THEOREM 4 (GENERALIZED ERDÖS-SZEKERES THEOREM). Given positive integers $d, k, n$ such that $\lceil d / 2\rceil+1 \leq k \leq d$, there exists an integer $n_{0}=E S_{d}(n, k)$ such that any set of $n_{0}$ points in $\mathbb{R}^{d}$ contains a subset $P$ of size $n$ with the following property: the simplex spanned by every $(d+1)-k$ points of $P$ lies on the boundary of $\operatorname{conv}(P)$. This statement is optimal, in the sense that this is not true for $k<\lceil d / 2\rceil+1$ for arbitrarily large pointsets.

The case $k=d$ simply corresponds to the Erdös-Szekeres theorem (that any large-enough set contains a lot of points in convex 
position). Of course the 'large-enough' size for the above theorem increases with decreasing $k$; but if one pays that price, one can get more properties. For example, for $d=4, k=3$, any large-enough set of points in $\mathbb{R}^{4}$ contains a large subset $Q$ where every edge spanned by points of $Q$ lies on $\operatorname{conv}(Q)$.

We now observe that this immediately carries over to an at-first nonobvious extension of Radon's theorem: if one is willing to increase the number of points, then a better upper-bound can be achieved on the sizes of the Radon partition:

THEOREM 1.1. Given an integer $\lfloor d / 2\rfloor+1 \leq k \leq d$, any set $P$ of $E S_{d}(d+2, k)$ points in $\mathbb{R}^{d}$ contains two sets $P_{1}, P_{2}$ such that $\operatorname{conv}\left(P_{1}\right) \cap \operatorname{conv}\left(P_{2}\right) \neq \emptyset$ and additionally, $\left|P_{1}\right|,\left|P_{2}\right| \leq k$. Furthermore, this is optimal in the sense that the statement does not hold for $k \leq\lfloor d / 2\rfloor$.

Proof. Apply Theorem 4 to $P$ to get a set of $d+2$ points $P^{\prime}$. Apply Radon's theorem to $P^{\prime}$ to get a partition $P_{1}, P_{2} \subset P^{\prime}$ whose convex hulls intersect. Now note that if $\left|P_{1}\right|>k$, then $\left|P_{2}\right| \leq(d+1)-k$. But then $\operatorname{conv}\left(P_{2}\right)$ lies on the convex hull of $P^{\prime}$, and so cannot intersect $\operatorname{conv}\left(P_{1}\right)$, a contradiction.

Optimality is obvious as $|P| \geq d+2$ for such a partition to exist (for $P$ in general position), and so one set has to have at least $\lfloor d / 2\rfloor+1$ points.

Our first result is to show that a similar extension is possible for Carathéodory's theorem (Section 4):

THEOREM 1.2. Given a set $P$ of $n$ points in $\mathbb{R}^{d}$ and a convex object $C$, either $P$ can be separated from $C$ by $O\left(d^{4} \log d\right)$ hyperplanes (i.e., each $p \in P$ is separated from $C$ by one of the hyperplanes), or $C$ intersects the convex hull of some $\lfloor d / 2\rfloor+1$-sized subset of $P$.

We show the above result by relating this problem to another well-known problem; in fact we prove that the bounds for these two problems are within a factor of $d$ of each other, a result of independent interest.

Unfortunately the above approach does not work for proving an extension of the colorful Carathéodory theorem, for which we give a proof using a different technique (Section 5):

THEOREM 1.3. For any $d$, there exists a constant $N_{d}$ such that given $k=\lfloor d / 2\rfloor+1$ sets of points $P_{1}, \ldots, P_{k}$ in $\mathbb{R}^{d}$ and a convex object $C$, either one of the sets $P_{i}$ can be separated from $C$ by $N_{d}$ hyperplanes, or there is a rainbow set of size $k$ whose convex hull intersects $C$.

Remark 1: Unlike the small polynomial bound in the extension of Carathéodory's theorem, the constant $N_{d}$ is exponential in $d$. We leave improving $N_{d}$ as an open problem.

Remark 2: The case where there are $d+1$ sets, and a set can be separated by one hyperplane is exactly the colorful Carathéodory theorem.

Remark 3: Note also that, as before, Theorem 1.3 implies the corresponding extension for Carathéodory's theorem, although with much worse quantitative bound than that given in Theorem 1.2.

Remark 4: Theorem 1.3 implies that given $C$ and a set of red and blue points in $\mathbb{R}^{3}$, either the red set or the blue set can be separated from $C$ by a constant $N_{3}$ number of planes. Or there is a red-blue edge intersecting $C$. In fact, for this case, a direct elementary proof with better constant exists, as follows. First, elementary considerations show that given a pointset and a convex set $C$, either there is a triangle $\Delta$ spanned by the points so that each edge of $\Delta$ intersects $C$ or all the point can be separated from $C$ using twelve hyperplanes. Now suppose that we have some red and blue points and a convex set $C$. Then applying the above result to each set of points, we conclude that either one of the sets can be separated from $C$ with twelve planes or there is a red triangle and a blue triangle each of whose edges intersect $C$. For each vertex of these triangles consider the region on the boundary of $C$ that it can see (considering $C$ to be opaque). Since each red (resp. blue) edge intersects $C$, no two of the red (resp. blue) regions intersect, i.e., no three of the six regions intersect at a common point. Since the regions are pseudodisks, their intersection graph is planar. As $K_{3,3}$ is not planar, there is a red region and a blue region which do not intersect. This implies that the red-blue edge defined by the points corresponding to these regions intersects $C$.

We conclude with some open problems and future directions of research in Section 6.

\section{MOTIVATION \& RELATED PROBLEMS}

Besides building on some basic theorems of discrete geometry, there are a few other reasons why statements of the type considered in this paper are useful.

\section{Weak $\epsilon$-nets.}

The weak $\epsilon$-net problem asks, given a set $P$ of $n$ points in $\mathbb{R}^{d}$, for the existence of a small-sized set $Q \subseteq \mathbb{R}^{d}$ such that any convex set containing at least $\epsilon n$ points of $P$ contains a point of $Q$. The current best upper-bound on the size of $Q$ required is $\tilde{O}\left(1 / \epsilon^{d}\right)$, while the best lower-bound is $\Omega\left(1 / \epsilon \log ^{d-1} 1 / \epsilon\right)$. It is not clear what upper-bound to expect. But if one believes that "the truth is probably much smaller, maybe around $O\left(1 / \epsilon^{\lfloor d / 2\rfloor}\right)$ " [Mat99], then consider the following natural approach from [MR08].

Pick a strong $\epsilon$-net $Q$ (with respect to the intersection of some constant $k$ halfspaces). Now suppose a convex object $C$ containing $\epsilon n$ points of $P$ is not hit by this strong $\epsilon$-net. Then it must be that no set of $k$ halfspaces separate $C$ from points of $Q$; otherwise the intersection of these halfspaces contains $C$ and so contains at least $\epsilon n$ points without containing any point of $Q$, a contradiction. Intuitively, this means that the points of $Q$ are 'close' to the boundary of $C$. It seems likely that then $C$ must contain a large fraction of the volume (w.r.t. some measure) of some low-dimensional simplex spanned by points of $Q$ (in which case we are done by picking weak nets of constant size within each low dimensional simplex). Unfortunately even the existence of an intersection of some lowdimensional simplex with $C$ is not clear. The best one can hope for is a large intersection with a $\lfloor d / 2\rfloor$-dimensional simplex (better than this is not possible - to see this consider $P$ to be the vertex set of a cyclic polytope and let $C$ to be a slightly shrunk copy of the polytope). This is somewhat similar to the inductive approach in [ABFK92, $\mathrm{CEG}^{+}$93]. Hence the above discussion provides one reason to study the separation interaction of low-dimensional simplices spanned by some set $P$ with a convex set $C$.

\section{High-depth edge.}

The following is a well-known problem open for more than 20 years: given a set $P$ of $n$ points in the plane, show that there exist two points of $P$ such that any disk containing them contains at least $n / 4$ points of $P$. The current-best bound is $n / 4.73$ [EHSS89]. We now show a close relation to the problems we study in this paper.

Given $P$, map $P$ to $P^{\prime}$ via a standard lifting to the paraboloid in 
$\mathbb{R}^{3}$. So each disk in $\mathbb{R}^{2}$ corresponds to a downward-facing halfspace in $\mathbb{R}^{3}$. Now let $C$ be the centerpoint region of $P^{\prime}$. By definition, each plane supporting a facet of $C$ has less than $n / 4$ points on the side not containing $C$. Now our goal is to find two points, say $p_{1}, p_{2}$ of $P^{\prime}$ such that any downward-facing halfspace containing $p_{1} p_{2}$ must contain at least one point of $C$ - and so contain at least $n / 4$ points. For contradiction, assume that for every pair of points, the "slab" swept by projecting the segment $p_{1} p_{2}$ onto the $x y$-plane avoids $C$. Then one would prove the conjecture by showing that there exist 4 planes supporting facets of $C$ that separate all the points of $P^{\prime}$ from $C$. This would yield a contradiction, as each such halfspace contains less than $n / 4$ points.

Therefore, the goal is to show that either the vertical slab for a pair intersects $C$, or all the points of $P^{\prime}$ can be separated by 4 supporting hyperplanes. Then the easier question of separating $P^{\prime}$ from $C$ with any separating hyperplanes if no edge intersects is exactly the extension of Carathéodory's theorem for $\mathbb{R}^{3}$.

\section{Gallai-type problems for Pseudodisks.}

Danzer proved that given any set of caps of a sphere in three dimensions such that every pair of them intersect, it is possible to pierce all of them using four points. While at first sight this appears unrelated to the problems we study, it is intimately related. Consider a sphere $S$ and a set of points in $\mathbb{R}^{3}$ outside it. For each point $p$, the set of points $x \in S$ such that a tangent plane to the sphere at $x$ separates $S$ from $p$ is a cap of $S$. Two points $p$ and $q$ can be separated from $S$ using a single plane iff their corresponding regions intersect. Danzer's theorem therefore implies that either the set of points can be separated from $S$ using four planes or there is an edge spanned by the points that intersects $S$. If we replace this sphere in $\mathbb{R}^{3}$ with an arbitrary convex set, then caps are replaced by regions that are pseudodisks. Danzer's result is believed to hold for pseudodisks as well but is currently known only with worse bounds. Therefore such separation problems can be seen as generalized Gallai-type problems.

\section{PRELIMINARIES}

In this section, we describe some basic results in discrete geometry that we will need.

Hadwiger-Debrunner ( $\boldsymbol{p}, \boldsymbol{q})$-theorem [AK92]. Given a set $S$ of convex sets in $\mathbb{R}^{d}$ such that out of every $p \geq d+1$ sets, there is a point common to $q \geq d+1$ of them, then $S$ has a hitting set of finite size and the minimum size of such a set is denoted by $H D_{d}(p, q)$ (so this is independent of $|S|$ ).

$\epsilon$-nets w.r.t. halfspaces in $\mathbb{R}^{d}$ [HW87]. Given a set $P$ of $n$ points in $\mathbb{R}^{d}$ and a parameter $\epsilon>0$, a set $Q \subseteq P$ is an $\epsilon$-net w.r.t. halfspaces if any halfspace containing at least $\epsilon n$ points of $P$ contains a point of $Q$. A famous theorem of Haussler-Welzl [HW87] shows that $\epsilon$-nets of size $O(d / \epsilon \log d / \epsilon)$ exist, independent of $n$. This bound was later improved in [KPW92] to a near-optimal bound of $(1+o(1))\left(\frac{d}{\epsilon} \log (1 / \epsilon)\right)$.

Centerpoint depth [Mat02]. Given any set $P$ of $n$ points in $\mathbb{R}^{d}$, the Tukey depth of a point $q \in \mathbb{R}^{d}$ is the minimum number of points of $P$ contained in any halfspace containing $q$. It is known that there always exists a point of Tukey depth at least $n /(d+1)$.

In general, the set of points of Tukey depth at least $\beta n$ form a convex region called the $\beta$-deep region of $P$. The $\beta$-deep region is non-empty for any $\beta \leq 1 /(d+1)$. It is the intersection of all halfspaces containing more than $(1-\beta) n$ points of $P$. Each facet of this region is supported by a hyperplane that passes through $d$ points of $P$. We will need the following fact:

Fact 1 [PA95]: If $P$ is a set of $n$ points and $h$ is a hyperplane defining a facet of the $\beta$-deep region $C$ of $P$, then the halfspace defined by $h$ that does not intersect the interior of $C$ contains less than $\beta n$ points of $P$.

\section{PROOF OF EXTENDED CARATHÉODORY'S THEOREM}

We first show that this problem is related to another problem involving low-dimensional simplices.

Let $f(d)$ be the smallest positive number such that for any set $P$ of points in $\mathbb{R}^{d}$, there exists a $\lfloor d / 2\rfloor+1$-sized subset $P^{\prime} \subseteq P$ such that any halfspace containing $P^{\prime}$ contains at least $|P| / f(\overline{(d)}$ points of $P$.

Let $g(d)$ be the smallest positive number such that given any set $P$ of points in $\mathbb{R}^{d}$ and a convex set $C$, if $P$ cannot be separated from $C$ using at most $g(d)$ hyperplanes, then $C$ must intersect the convex hull of some $\lfloor d / 2\rfloor+1$ size subset of $P$.

We now show that $g(d)$ and $f(d)$ are related within a factor of $d$.

THEOREM 4.1. $g(d) \leq d \cdot f(d) \log f(d)$. In other words, given a set $P$ of points in $\mathbb{R}^{d}$ and a convex set $C$ such that $P$ cannot be separated from $C$ by $d f(d) \log f(d)$ hyperplanes, then $C$ must intersect the convex hull of some $\lfloor d / 2\rfloor+1$ points of $P$.

Proof. Assume that no convex hull of any $\lfloor d / 2\rfloor+1$ points of $P$ intersects $C$. Then we show that $P$ can be separated from $C$ using $d f(d) \log f(d)$ hyperplanes.

ClAIM 4.2. Let $P$ be a weighted set of points in $\mathbb{R}^{d}$, with weight of the point $p_{i} \in P$ to be $w_{i}$. Assume all $w_{i}$ 's are rationals, and let $W=\sum w_{i}$. If the convex hull of no $\lfloor d / 2\rfloor+1$ points of $P$ intersects $C$, then there exists a hyperplane separating points of total weight at least $\frac{W}{f(d)}$ from $C$.

Proof. As each $w_{i}$ is a rational, assume $w_{i}=\hat{w}_{i} / D$, where $\hat{w}_{i}$ and $D$ are integers. Let $Q$ be the pointset gotten by replacing each point $p_{i}$ with $\hat{w}_{i}$ copies of $p_{i}$. Crucially, if the convex hull of no $\lfloor d / 2\rfloor+1$ subset of $P$ intersects $C$, then the convex hull of no $\lfloor d / 2\rfloor+1$ subset of $Q$ can intersect $C$. Take the $\lfloor d / 2\rfloor+1$-sized subset $Q^{\prime}$ of $Q$ such that any halfspace containing $Q^{\prime}$ contains at least $|Q| / f(d)$ points of $Q$. As the convex hull of $Q^{\prime}$ does not intersect $C$, there is a halfspace $h$ which does not intersect $C$ and contains $Q^{\prime}$. Let $P^{\prime}$ be the set of points of $P$ contained in $h$. Then $h$ contains exactly $\sum_{p_{i} \in P^{\prime}} \hat{w}_{i}$ points of $Q$, which by definition of $Q^{\prime}$ must be at least $|Q| / f(d)$. Then the sum of weights of points of $P$ contained in $h$ is bounded by

$$
\begin{aligned}
\sum_{p_{i} \in P^{\prime}} w_{i} & =\frac{\sum_{p_{i} \in P^{\prime}} \hat{w}_{i}}{D} \geq \frac{|Q| / f(d)}{D}=\frac{\left(\sum \hat{w}_{i}\right) / f(d)}{D} \\
& =\frac{\sum w_{i} D}{D f(d)}=\frac{W}{f(d)}
\end{aligned}
$$

Discretize the set of all combinatorially distinct hyperplanes separating some subset of $P$ from $C$ to get a set $\mathcal{H}=\left\{h_{1}, \ldots, h_{m}\right\}$ of $O\left(|P|^{d}\right)$ hyperplanes. Now consider assigning weights $w\left(h_{i}\right)$ to each halfspace such that the total weight $\sum w\left(h_{i}\right)$ is minimized, and the sum of weights of halfspaces containing any point of $P$ is at least 1 . Let $W(\mathcal{H})$ denote the minimum value.

Similarly, assign weights $w\left(p_{i}\right)$ to each point of $P$ such that the total weight $\sum w\left(p_{i}\right)$ is maximized, and the sum of weights of 
points contained in any halfspace $h \in \mathcal{H}$ is at most 1 . Let $W(P)$ denote the maximum value. Then the above two problems are dual to each other (as linear programs), and so by the Strong Duality Theorem, $W(\mathcal{H})=W(P)$.

Now note that $W(P) \leq f(d)$ : by Claim 4.2, there exists a halfspace in $\mathcal{H}$ of weight at least $W(P) / f(d)$, which by the definition of $W(P)$ is at most 1 .

Therefore there exists an assignment of weights to halfspaces in $\mathcal{H}$ such that $W(\mathcal{H}) \leq f(d)$, and each point is contained in halfspaces of total weight at least 1 . Now using the $\epsilon$-net theorem for halfspaces [KPW92], with $\epsilon=1 / W(\mathcal{H})$, there exists a set of

$$
d / \epsilon \log 1 / \epsilon=d W(\mathcal{H}) \log W(\mathcal{H})=d f(d) \log f(d)
$$

halfspaces of $\mathcal{H}$ containing all points of $P$. As all halfspaces in $\mathcal{H}$ were separating halfspaces, we are done.

Remark 1: The above technique is similar to the one used in the proof of Hadwiger-Debrunner $(p, q)$ theorem [AK92], with some crucial differences. In their use, they get an exponential bound, which we are able to avoid due to three reasons: $\epsilon$-nets for halfspaces have a near-linear bound, avoiding double-counting arguments that they use, and finally, the weighted version (Claim 4.2) gives exactly the same quantitative bound as the unweighted version.

Proof of Theorem 1.2: the paper [SSW08] proves that $f(d) \leq$ $O\left(d^{3}\right)$. And the proof is complete by using Theorem 4.1.

Similarly we now show that a bound on $g(d)$ gives an upperbound on $f(d)$ :

TheOREM 4.3. $f(d) \leq d \cdot g(d)$. In other words, given a set $P$ of points in $\mathbb{R}^{d}$, there always exists a subset $P^{\prime}$ of size $\lfloor d / 2\rfloor+1$ such that any halfspace containing $P^{\prime}$ contains at least $|P| / d g(d)$ points of $P$.

Proof. Consider the $\beta$-deep region $C$ of $P$; by the Centerpoint theorem, for $\beta \leq 1 /(d+1)$, such a region always exists. Now we claim that for $\beta=1 / d g(d)$, there exists a $\lfloor d / 2\rfloor+1$-sized subset $P^{\prime}$ whose convex hull intersects $C$. Then any halfspace containing $P^{\prime}$ contains at least one point of $C$, and so contains at least $|P| / d g(d)$ points by the definition of the centerpoint region.

Otherwise, for contradiction assume that the convex hull of no $\lfloor d / 2\rfloor+1$-sized subset intersects $C$. Then by definition of $g(d), P$ can be separated from $C$ using $g(d)$ hyperplanes, say the set $\mathcal{H}$.

Now any halfspace not intersecting $C$ contains less than $d \cdot \beta|P|$ points: each halfspace supporting a facet of $C$ contains less than $\beta|P|$ points, and any other halfspace not intersecting $C$ is contained in the union of at most $d$ halfspaces supported by facets of $C$.

Therefore each halfspace of $\mathcal{H}$ contains less than $d \cdot \beta|P|$ points. And so the union of halfspaces in $\mathcal{H}$ contains less than $g(d) \cdot d \cdot \beta|P|$ points of $P$, a contradiction for $\beta=1 / d g(d)$.

\section{PROOF OF EXTENDED COLORFUL CARATHÉODORY THEOREM}

The goal of this section is to prove Theorem 1.3. We use a slightly different language for convenience: instead of saying that "a point set $P$ can be separated from a convex body $C$ using $k$ hyperplanes", we say that "there exists a polyhedron $\mathcal{Q}$ with $k$ facets such that $C \subseteq Q$ and $Q \cap P=\emptyset$ ". In such a case we also say that $\mathcal{Q}$ separates $P$ from $C$. We re-state Theorem 1.3 in this language.

THEOREM 5. For any positive $d$ and $l>\lfloor d / 2\rfloor$, there exists a constant $N_{d, l}$ s.t. the following is true. Given any compact convex body $C$ and l finite sets of points $P_{1}, \ldots P_{l}$ in $\mathbb{R}^{d}$, at least one of the following holds:
1. There exists a polyhedron $\mathcal{Q}$ with at most $N_{d, l}$ facets such that for some $i, \mathcal{Q}$ separates $P_{i}$ from $C$.

2. There exists a rainbow subset $P^{\prime} \subseteq \cup_{i=1}^{d} P_{i}$ whose convex hull intersects the interior of $C$.

The approach of the previous section does not work for proving the extension of the colorful Carathéodory's theorem. The method gave one low-dimensional simplex intersecting $C$, or the existence of a hyperplane separating many points. Unfortunately with multiple sets, one needs to find many such intersecting simplices for each set $P_{i}$. Therefore one is forced to use a more Ramsey-theoretic technique, and this causes the constant to become exponential in $d$.

We next present some preliminary definitions and claims required to prove the above theorem.

Call a convex body $C$ fine if it is compact and its boundary $\partial C$ is smooth and has positive curvature everywhere. Let $C$ be a fine convex body and let $P$ be a finite set of points in $\mathbb{R}^{d}$. We say that a point $p$ can see a point $y$ if the relative interior of the segment $p y$ does not intersect $C$. For any $p \in P$, let $U_{p}$ be the set of points in $\partial C$ that $p$ can see.

Let $h_{y}$ be the tangent plane to $C$ at the point $y \in \partial C$ and let $h_{y}^{+}$ be the closed halfspace defined by it that contains $C$. Observe that any point $p \in P$ sees a point $y \in \partial C$ iff $p \notin \operatorname{int}\left(h_{y}^{+}\right)$, where $\operatorname{int}(S)$ denotes the interior of the set $S$.

LEMMA 5.1. For any positive numbers $d$ and $t \geq d$, there exists a constant $H_{t, d}$ such that given any fine convex body $C$ and a finite set of points $P$ such that $P \cap C=\emptyset$, at least one of the following hold:

- There exists a set $X \subset \partial C$ of size at most $H_{t, d}$ such that each point in $P$ is seen by some $x \in X$.

- There is a subset $P^{\prime} \subseteq P$ of size at least $t$ such that no $y \in \partial C$ sees more than $d-1$ points in $P^{\prime}$.

PROOF. Fix any point $\nu \in \partial C$ as a reference point. Let $h_{\nu}$ be the tangent plane to $C$ at $\nu$. Let $h$ be the unique tangent hyperplane parallel to $h_{\nu}$ such that $C$ is contained in the strip between $h_{\nu}$ and $h$. Let $\pi$ be the continuous bijective map that maps any $y \in \partial C \backslash$ $\{\nu\}$ to $l(\nu, y) \cap h$, where $l(\nu, y)$ denotes the line through $\nu$ and $y$.

For any $p \in P$ that does not see $\nu$ (i.e., $\nu \notin U_{p}$ ), let $V_{p}=$ $\pi\left(U_{p}\right)=\left\{\pi(y): y \in U_{p}\right\}$ and let $\mathcal{V}=\left\{V_{p}: p \in P, p\right.$ does not see $\left.\nu\right\}$. Clearly, for each $p \in P$ that does not see $\nu, V_{p}$ lies on the plane $h$, is convex and has dimension $d-1$. Note that if two points $p, q \in P$ see a point $y \in \partial C$ then $y \in U_{p} \cap U_{q}$. So $\pi(y) \in V_{p} \cap V_{q}$.

Suppose that the second part of the theorem does not hold; i.e., in every subset of $P$ of size $t$, there are at least $d$ points which can be seen by a single point $y \in \partial C$. Equivalently, any subset of $\mathcal{V}$ of size $t$ has at least $d$ sets which have a common intersection. By the Hadwiger-Debrunner theorem [AK92], there exists a constant $H D_{d-1}(t, d)$ of points in $h$ that hit all the sets in $\mathcal{V}$. Let $X^{\prime}$ be the set of these points. Let $X=\pi^{-1}\left(X^{\prime}\right) \cup\{\nu\}$. Each point in $P$ is seen by at least one point in $X$ (if $V_{p}$ is hit by the point $y^{\prime} \in h$, then $p \in P$ is seen by $\left.\pi^{-1}\left(y^{\prime}\right) \in \partial C\right)$. The theorem is therefore proved by setting $H_{t, d}=H D_{d-1}(t, d)+1$.

Now we can finish the proof of the main theorem of this section:

Proof. Let $P=\cup_{i=1}^{l} P_{i}$. We set $N_{d, l}=H_{t, d}$ for some $t$ to be fixed later

If $P \cap C \neq \emptyset$ then the second part of the theorem is trivially satisfied. We therefore assume that $P \cap C=\emptyset$. Without loss of generality we also assume that $C$ is fine since we can always find a 
fine convex body $C^{\prime}$ that contains $C$ and does not intersect $P$ and furthermore for each point $y^{\prime} \in C^{\prime}$, there is a point $y \in C$ such that the Euclidean distance between $y$ and $y^{\prime}$ is smaller than any prescribed $\delta>0$. Proving the theorem for such $C^{\prime}$ s also proves it for arbitrary closed convex bodies.

For each $i$, apply Lemma 5.1 to $C$ and $P_{i}$ with the parameter $t$. This gives us either a set $X_{i}$ of at most $H_{t, d}$ points in $\partial C$ such that each point in $P_{i}$ is seen by at least one of these or we get a set $Q_{i} \subseteq P_{i}$ of $t$ points so that no $d$ of them is seen by the same point in $\partial C$. If the first possibility happens for some $j$, then $\bigcap_{x \in X_{j}} h_{x}^{+}$ gives us the polyhedron $\mathcal{Q}$ with at most $H_{t, d}$ facets and where $\mathcal{Q}$ contains $C$ while $P_{j}$ lies outside $\mathcal{Q}$. This satisfies the first part of the Theorem and we're done.

We therefore assume the second possibility for each $i$; namely, each $P_{i}$ has a subset $Q_{i}$ of $t$ points such that no $d$ points of $Q_{i}$ are seen by the same point of $\partial C$. Equivalently, the convex hull of any $d$ points of $Q_{i}$ intersects $C$.

Let $Q=\cup_{i=1}^{l} Q_{i}$. Consider any rainbow set $R \subseteq Q$ with one point from each $Q_{i}$. There are $t^{l}$ such sets. If the convex hull of $R$ intersects $C$, then the second part of the theorem is satisfied, and we're done. Assume for contradiction that this is not the case. Then for each rainbow set $R$, there exists a hyperplane $h$ separating $R$ from $C$. The closed halfspace $h^{-}$bounded by $h$ and not intersecting $C$ contains at most $d-1$ points from any particular $Q_{i}$ due to the fact that any $d$-sized subset of $Q_{i}$ intersects $C$. Therefore $\left|h^{-} \cap Q\right| \leq(d-1) l$, and hence is a $\leq k$-set of $Q$ with $k=l(d-1)$.

If no rainbow set intersects $C$, then we get such a $\leq k$-set for each rainbow set $R$ of size $l$. As there are $t^{l}$ such rainbow sets, we get $t^{l} \leq k$-sets. However each such $\leq k$-set can be overcounted at most $\left(\begin{array}{l}k \\ l\end{array}\right)=\left(\begin{array}{c}l(d-1) \\ l\end{array}\right)$ times. This implies that there are at least $L(t)=t^{l} /\left(\begin{array}{c}l(d-1) \\ l\end{array}\right)$ distinct $\leq k$-sets. On the other hand, it is known that the number of $<k$-sets of a set of $n$ points in $\mathbb{R}^{d}$ is at most $O\left(n^{\lfloor d / 2\rfloor}(k+1)^{\lceil d / 2\rceil}\right)$ [Mat02]. This gives an upper bound of $U(t)=O\left((t l)^{\lfloor d / 2\rfloor}((d-1) l+1)^{\lceil d / 2\rceil}\right)$ on the number of $\leq k$ sets. Since $l>\lfloor d / 2\rfloor$, for some large enough $t$ depending only on $l$ and $d, L(t)>U(t)$. Thus we get a contradiction implying that one of the rainbow sets must intersect $C$.

\section{CONCLUSIONS}

We think that the phenomenon studied in this paper is much more widely applicable. In fact, statements of this type might shed light on interactions between several classical theorems (see below). Besides improving quantitative bounds presented in this paper - for both extended Carathéodory and the extended colorful Carathéodory theorems - we end with a number of questions for which we think the answer is affirmative:

1. Extension of Kirchberger's theorem. Given a set $P$ of $n$ red points and $n$ blue points in $\mathbb{R}^{d}$ either there exist a constant number of hyperplanes $\mathcal{H}$ such that every red-blue pair is separated by a plane in $\mathcal{H}$, or a $\lfloor d / 2\rfloor$-dimensional red simplex intersects a $\lfloor d / 2\rfloor$-dimensional blue simplex.

2. Extension of Tverberg's theorem. Given a set $P$ of $n$ points in $\mathbb{R}^{d}$, there exists a Tverberg partition on a large subset of $P$ where two sets have size $\lfloor d / 2\rfloor+1$. It is not too hard to see that this implies a version of the extension of Carathéodory's theorem.

3. Extension of Colored Tverberg's theorem. Given $(d+$ $2)$ sets $P_{1}, \ldots, P_{d+2}$, there exists a colored Tverberg partition on a large subset where two sets have size $\lfloor d / 2\rfloor+$ 1. Again, this version implies the extension of the colorful Carathéodory theorem.

\section{ACKNOWLEDGEMENTS}

We thank an anonymous reviewer for meticulous and very insightful comments. The first author would also like to thank Janos Pach for his support and encouragement. Part of this work was done by the first author while visiting the wonderful algorithms group at the Max-Planck Institute for Informatik in Saarbrucken.

\section{References}

[ABFK92] Noga Alon, Imre Bárány, Zoltán Füredi, and Daniel J. Kleitman. Point selections and weak $\varepsilon$-nets for convex hulls. Combinatorics, Probability \& Computing, 1:189-200, 1992.

[AK92] N. Alon and D. Kleitman. Piercing convex sets and the Hadwiger Debrunner (p; q)-problem. Adv. Math., 96(1):103-112, 1992.

[Bár82] Imre Bárány. A generalization of Carathéodory's theorem. Discrete Mathematics, 40(2-3):141-152, 1982.

[CEG $\left.{ }^{+} 93\right]$ Bernard Chazelle, Herbert Edelsbrunner, Michelangelo Grigni, Leonidas Guibas, Micha Sharir, and Emo Welzl. Improved bounds on weak $\varepsilon$-nets for convex sets. In Proceedings of the twenty-fifth annual ACM Symposium on Theory of Computing, pages 495-504, 1993.

[EHSS89] H. Edelsbrunner, N. Hasan, R. Seidel, and X.J. Shen. Circles through two points that always enclose many points. Geom. Dedicata, 32:1-12, 1989.

[HW87] D. Haussler and E. Welzl. Epsilon-nets and simplex range queries. Discrete Comput. Geom., 2:127-151, 1987.

[KPW92] J. Komos, J. Pach, and G. Woeginger. Almost tight bounds for epsilon nets. Discrete \& Computational Geometry, pages 163-173, 1992.

[Mat99] J. Matousek. Piercing and selection theorems in convexity, 1999.

[Mat02] Jirí Matousek. Lectures in Discrete Geometry. SpringerVerlag, New York, NY, 2002.

[MR08] N. Mustafa and S. Ray. Weak $\epsilon$-nets have a basis of size $\mathrm{O}(1 / \epsilon \log 1 / \epsilon)$. Comp. Geom: Theory and Appl., 40(1):84$91,2008$.

[PA95] Janos Pach and Pankaj K. Agarwal. Combinatorial Geometry. John Wiley \& Sons, New York, NY, 1995.

[Rad47] R. Rado. A theorem on general measure. J. London. Math. Soc., 21:291-300, 1947.

[SSW08] Shakhar Smorodinsky, Marek Sulovský, and Uli Wagner. On center regions and balls containing many points. In Proceedings of the 14th annual International Conference on Computing and Combinatorics, COCOON'08, pages 363-373, 2008. 